\title{
High intensity repeated sprints impair postural control, but with no effects on free throwing accuracy, in under-19 basketball players
}

\author{
Fabio Augusto Barbieri ${ }^{\mathrm{a}, *}$, Sérgio Tosi Rodrigues ${ }^{\mathrm{a}}$, Paula Favaro Polastri ${ }^{\mathrm{a}}$, \\ Ricardo Augusto Barbieri ${ }^{\text {b,c }}$, Pedro Henrique Alves de Paula ${ }^{a}$, Fabio Milioni ${ }^{a}$, \\ Paulo Eduardo Redkva ${ }^{a}$, Alessandro Moura Zagatto ${ }^{a}$
}

a São Paulo State University (UNESP) - Human Movement Research Laboratory (MOVI-LAB), Laboratory of Physiology and Human Performance (LAFIDE), Laboratory of Information, Vision and Action (LIVIA) - School of Sciences, Department of Physical Education, Bauru, São Paulo, Brazil

b School of Physical Education and Sport of Ribeirão Preto, University of São Paulo - USP, (GECIFEX - Outlier-Lab), Ribeirão Preto, Brazil

c Centro Universitário Estácio de Ribeirão Preto, Brazil

\section{Introduction}

High intensity exercise is an intervenient aspect of postural control. After high intensity exercise, athletes present decreased postural control performance (Steinberg et al., 2016), which is evident in the form of increased body sway (Pau, Ibba, \& Attene, 2013; Zemková \& Hamar, 2014). Moreover, high intensity exercise leading to fatigue can cause an increase in the risk of injuries (Hebert, Corboy, Manago, \& Schenkman, 2011; Steinberg et al., 2015). However, athletes who present better performances in repeated sprints (RSA), which is an all-out exercise, present less impairment in balance control, especially in the single-legged stance (Pau et al., 2013). In addition, improved general aerobic fitness attenuates the negative effects of fatigue on postural adjustments (Hebert et al., 2011) and better aerobic fitness improves body sway in non-fatigue conditions (Paillard, 2012; Steinberg et al., 2015). Specifically for basketball players, an adverse effect of high intensity exercise on passing accuracy in basketball has been shown, mainly in novice players when compared to experts (Lyons, Al-Nakeeb, \& Nevill, 2006). In addition, an increment in exercise intensity seems to decrease performance (accuracy) in free throwing (Padulo, Attene et al., 2015), but without significant effects on the kinematics of free throw shooting (Uygur, Goktepe, Ak, Karabörk, \& Korkusuz, 2010).

Postural control is considered one of the limiting factors of sport performance, involving multiple sensorimotor processes (Horak \& Macpherson, 2011; Zemková \& Hamar, 2014). Modulations in body sway are necessary when practicing sport skills to support high intensity exercises and improve postural control (Zemková \& Hamar, 2014). Increased postural instability seems to reduce performance (Zemková, 2009), principally in movements that requires accuracy, such as rifle shooting and archery (Mononen, Konttinen, Viitasalo, \& Era, 2007; Platzer, Raschner, \& Patterson, 2009). Accuracy in free throw shooting in basketball, which is a considerably decisive element in a basketball game (Uygur et al., 2010), has been related to low horizontal oscillation and high body stability (Hudson, 1985). Professional basketball players with better performance in shooting present higher postural control (Perrin, Perrin, Courant, Bene, \& Durupt, 1990). However, in previous studies postural control has been assessed during controlled situations, when the athletes did not perform a high intensity exercise before the shooting. Rapid readjustment of postural control after high intensity exercise is considered an important ability to improve basketball performance and avoid injuries (Steinberg et al., 2015, 2016; Zemková \& Hamar, 2014). Therefore, the question of this study is: What are the effects of high intensity exercise on body sway (postural control) and accuracy in free throw shooting in basketball players?

The aim of this study was to investigate the effects of high intensity exercise (all-out sprints) on body sway in under-19 basketball players during bipedal stance and to correlate body sway parameters and free throwing performance, and body sway parameters and

\footnotetext{
* Corresponding author at: Universidade Estadual Paulista - UNESP - FC - Bauru, Human Movement Research Laboratory (MOVI-LAB), Av. Eng. Luiz Edmundo Carrijo Coube, 14-01, CEP: 17033-360 Bauru, SP, Brazil.

E-mail address: barbieri@fc.unesp.br (F.A. Barbieri).
} 
performance in high intensity exercise. We hypothesized that high intensity exercise performed through an RSA protocol would increase body sway and there would be a negative relation between body sway parameters and performance and a positive relation between body sway and performance in high intensity exercise (higher performance with smaller body sway). The study of the effects of all-out exercise on body sway in basketball players is important for performance purposes, since deficits in postural control may decrease performance and increase the risk of injuries (Pau et al., 2013; Zemková \& Hamar, 2014).

\section{METHODS}

\subsection{Subjects}

Twenty-five male under-19 basketball players from a Brazilian team participated in the study (16- to 19-years old, height: $1.90 \pm 0.09 \mathrm{~m}$; body mass: $75.75 \pm 3.86 \mathrm{~kg}$, arm span: $1.97 \pm 0.96 \mathrm{~m}$ ). The athletes had at least three years basketball experience and participated in $12 \mathrm{~h}$ of training per week. The athletes were national level and the majority participated in training with the professional team. The inclusion criteria for the study were: i) having participated regularly in previous national competitive seasons and ii) being healthy (no chronic diseases and/or musculoskeletal injuries).

\subsection{Design}

Participants were required to abstain from alcohol or caffeinated beverages during the experimental period and eat at least $2-3 \mathrm{~h}$ prior to testing, to reduce any interference in the experiment. All tests were conducted on an indoor basketball court. The tests were performed in the same period of the day (from 16:00 to 20:00 $\mathrm{h}$ - regular training schedule) to eliminate any influence of the circadian cycle. The athletes were rested before the test. The study was approved by the local ethics committee. All participants and their parents/guardians signed an informed consent form.

\subsection{Methodology}

A force plate $-50 \times 50 \mathrm{~cm}$ (AccuGait, Advanced Mechanical Technologies, Boston, MA, USA), with a rate of 100 samples/s, was used to analyse postural control. Body sway was measured before and after a high intensity exercise (RSA) protocol (Fig. 1). The evaluation of body sway after the RSA protocol was performed after free throws without a rest period. Each participant performed three trials of $30 \mathrm{~s}$ each in a quiet stance (due to the protocol design, short rest time between the sprints $-30 \mathrm{~s}$, it was not possible to perform more time in a quiet stance for each trial). Participants were instructed to: i) position their feet parallel to each other (placed at a similar distance from the pelvis and the position was maintained in each trial); ii) stand quietly in an upright position; iii) keep their eyes open and direct their gaze to the front (an experienced researcher ensured that the participants remained quiet with their gaze to the front). The three force and moment components were acquired in the vertical, anterior-posterior, and medio-lateral directions. The mean center of pressure (CoP) signal was calculated for each analysis. The first $10 \mathrm{~s}$ of each recording was ignored systematically to avoid potential disturbances resulting from delayed stabilization after the participant stepped onto the force plate. The data were then filtered with a fourth order low-pass Butterworth filter with a cut-off frequency of $5 \mathrm{~Hz}$ determined by residual analysis (Winter, 2009).

The following parameters of the CoP in the anterior-posterior and medio-lateral directions were analysed: the total displacement of sway (i.e., the length of the CoP trajectory on the support base); the mean velocity of sway (i.e., the displacement of the total sway of the CoP divided by the total duration of the trial); the root mean square (RMS) of sway displacement (i.e., the CoP variability around the mean CoP trajectory); and spectral analysis of the position time series, separately in each direction, and used to calculate the median frequencies of the data (Matlab software version 7.10, Mathworks). In addition, the area of sway (i.e., the area of an ellipse containing $95 \%$ of the CoP data) was calculated.

\subsubsection{Repeated sprint ability test}

An RSA protocol was used to simulate high intensity exercise (all-out sprints) (Milioni, Redkva, Barbieri, \& Zagatto, 2017; Padulo, Laffaye et al., 2015). The test consisted of ten $30 \mathrm{~m}$ sprints with two $180^{\circ}$ changes of direction $(10 \mathrm{~m}+10 \mathrm{~m}+10 \mathrm{~m})$, interspaced by $30 \mathrm{~s}$ of passive recovery between the sprints (Padulo, Laffaye et al., 2015; Zagatto et al., 2017) and with an exercise-to-rest ratio of 1:5 (Ruscello et al., 2013) (test-retest Intraclass correlation > 0.90) (Padulo, Laffaye et al., 2015). Time of each sprint was recorded using photoelectric cells (Speed Test - Cefise ${ }^{\circledR}$ - Nova Odessa - SP - Brazil) positioned at the beginning and end of the track. Verbal encouragement was given during the sprints. Before and after the RSA protocol, the athletes performed a set of three free throws ( $4.6 \mathrm{~m}$ - free throw line distance from point on floor directly below backboard). Each set of three free throws was performed quickly $(\sim 10 \mathrm{~s})$ during the recovery period. The accuracy (number of hits) was measured for each set.

The best time (BT - best sprint time), mean time (MT), worst time (WT - worst sprint time), and percentage decrement in sprint

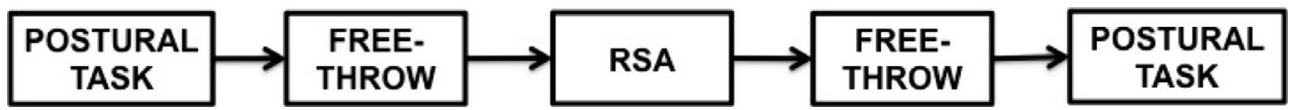

Fig. 1. Schematic of the sequence of protocols in the experiment. RSA - repeated sprints. 
Table 1

Descriptive data (mean \pm SD) of RSA parameters.

\begin{tabular}{ll}
\hline Parameter & Mean \pm SD \\
\hline TT (s) & $70.29 \pm 3.05$ \\
BT (s) & $6.75 \pm 0.27$ \\
MT (s) & $7.03 \pm 3.05$ \\
WT (s) & $7.34 \pm 0.41$ \\
\%Dec (\%) & $4.13 \pm 1.46$ \\
{$[\mathrm{La}]_{\text {PEAK }}\left(\mathrm{mmol.L}^{-1}\right)$} & $8.23 \pm 2.50$
\end{tabular}

Values are means \pm SD. TT $=$ Total Time (s). BT $=$ Best Time.

MT $=$ Mean Time. $\quad$ WT $=$ Worst $\quad$ Time. $\%$ Dec $=$ Decrease in

Percentage. $[\mathrm{La}]_{\mathrm{PEAK}}=$ Blood Lactate concentration.

times (\%Dec - percentage of decrement between best and worst sprint performance) were measured per Fitzsimons, Dawson, Ward, and Wilkinson (1993) [\%Dec $=100 \times($ sum of sprint times $/($ mean of sprint time $\times 10))-100)]$. Additionally, blood samples were taken from the earlobes in $25 \mu \mathrm{L}$ heparinized capillary tubes before and 3, 5, 7, and $10 \mathrm{~min}$ after the RSA protocol, to determine blood lactate concentrations using a lactate analyser (YSI 2300 Sport ${ }^{\circledR}$, Yellow Spring Instruments, Yellow Spring, OH, USA).

\subsection{Statistical analysis}

Statistical analyses were performed using SPSS software (version 18.0) for Windows (p $<0.05$ ). The data were normally distributed and the assumption of sphericity was not violated, as verified by the Shapiro-Wilk and Mauchly tests, respectively. Our primary outcomes, body sway parameters and accuracy, were analysed using the paired samples T-test (before high intensity exercise $\mathrm{x}$ after high intensity exercise). The effect size obtained in each statistical analysis is also presented and interpreted as proposed by Hopkins (www.sportsci.org/resource/stats), with effect size $<0.2$ considered as trivial, small between 0.2 and 0.5 , moderate between 0.6 and 1.1, large between 1.2 and 1.9, and very large $>2.0$. Finally, the Spearman correlation and Pearson correlation were calculated to verify the relation between accuracy and body sway parameters and between performance in high intensity exercise parameters (total time, best sprint time, meantime, worst sprint time, percentage of sprint performance decrement, and peak of blood lactate concentration) and body sway parameters, respectively, separately for variables before and after high intensity exercise.

\section{Results}

To illustrate the high intensity exercise parameters simulated by the RSA protocol, Table 1 shows the total time (TT), best sprint time (BT), mean time (MT) worst sprint time (WT), percentage of sprint performance decrement (\%Dec), and peak of blood lactate concentration ([La $\left.]_{\text {PEAK }}\right)$.

After high intensity exercise (Table 2), the basketball players presented increased anterior-posterior and medio-lateral displacement $\left(\mathrm{t}_{24}=-5.19\right.$ and $\mathrm{t}_{24}=-5.33$, respectively, $\left.\mathrm{p}<0.001\right)$, mean velocity $\left(\mathrm{t}_{24}=-8.33\right.$ and $\mathrm{t}_{24}=-7.60$, respectively, $\mathrm{p}<0.001$ ), and RMS $\left(\mathrm{t}_{24}=-5.17\right.$ and $\mathrm{t}_{24}=-4.76$, respectively, $\left.\mathrm{p}<0.001\right)$. In addition, the area of sway was higher after the RSA protocol $\left(\mathrm{t}_{24}=-4.76, \mathrm{p}<0.001\right)$. To illustrate the CoP behaviour before and after high intensity exercise, Fig. 2 shows a typical example of the time series of CoP displacement for each condition. For accuracy in free throws, the statistical analysis indicated a small effect size (0.51) and no difference between before (1.39 \pm 0.75 number of hits - median $=1$ hit $)$ and after

Table 2

Means and standard deviations of body sway parameters before and after high intensity exercise.

\begin{tabular}{|c|c|c|c|}
\hline & Before high intensity exercise & After high intensity exercise & Effect Size \\
\hline \multicolumn{4}{|l|}{ Displacement (cm) } \\
\hline Anterior-posterior & $189.18 \pm 63.33$ & $297.59 \pm 120.23^{*}$ & 1.18 (large effect) \\
\hline Medio-lateral & $374.05 \pm 97.57$ & $554.70 \pm 176.43^{*}$ & 1.32 (large effect) \\
\hline \multicolumn{4}{|l|}{ Mean velocity $(\mathrm{cm} / \mathrm{s})$} \\
\hline Anterior-posterior & $0.22 \pm 0.04$ & $0.33 \pm 0.07^{*}$ & 2.00 (large effect) \\
\hline Medio-lateral & $0.33 \pm 0.07$ & $0.55 \pm 0.15$ & 2.00 (large effect) \\
\hline \multicolumn{4}{|l|}{$R M S(\mathrm{~cm})$} \\
\hline Anterior-posterior & $0.10 \pm 0.03$ & $0.16 \pm 0.06^{*}$ & 1.33 (large effect) \\
\hline Medio-lateral & $0.19 \pm 0.04$ & $0.28 \pm 0.09^{*}$ & 1.38 (large effect) \\
\hline \multicolumn{4}{|l|}{ Frequency $(\mathrm{Hz})$} \\
\hline Anterior-posterior & $0.51 \pm 0.17$ & $0.49 \pm 0.16$ & -0.12 (small effect) \\
\hline Medio-lateral & $0.37 \pm 0.13$ & $0.38 \pm 0.12$ & 0.08 (small effect) \\
\hline Area $\left(\mathrm{cm}^{2}\right)$ & $0.35 \pm 0.16$ & $0.87 \pm 0.59^{*}$ & 0.39 (small effect) \\
\hline
\end{tabular}

* $\mathrm{p}<0.05$ compared to before high intensity exercise. 


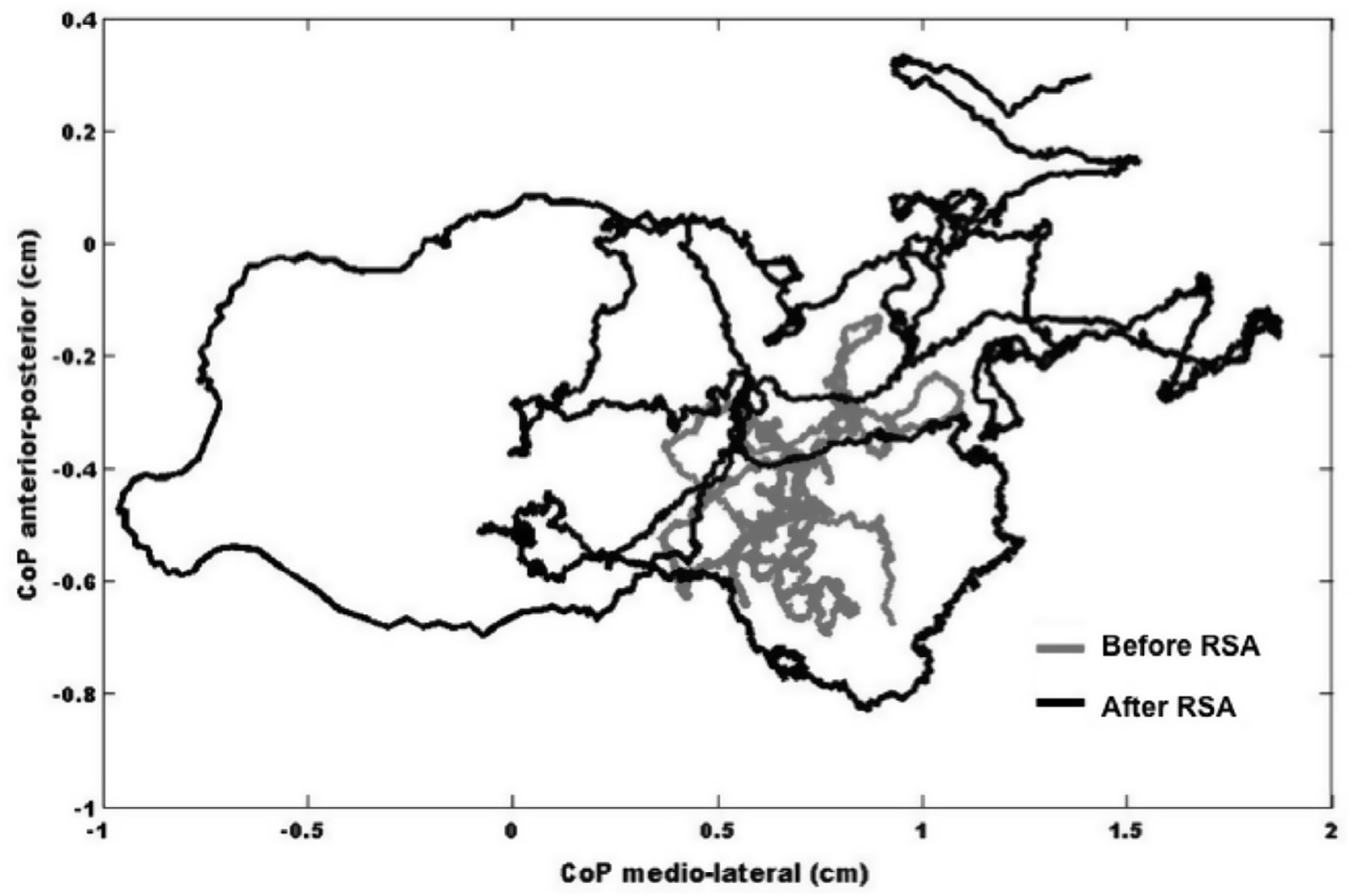

Fig. 2. Examples of the CoP displacement before and after high intensity exercise (RSA - repeated sprints) in the same subject.

$(1.79 \pm 0.83$ number of hits - median $=2$ hits $)$ high intensity exercise $(\mathrm{p}=0.08)$

There was a moderate inverse correlation between accuracy and medio-lateral mean velocity $(\mathrm{r}=-0.48, \mathrm{p}<0.01)$, mediolateral RMS ( $r=-0.43, \mathrm{p}<0.03)$, and area of body sway $(\mathrm{r}=-0.49, \mathrm{p}<0.01)$ before high intensity exercise. In addition, there was a moderate positive correlation between anterior-posterior displacement, velocity and RMS and medio-lateral mean velocity and total time $(\mathrm{r}=0.50, \mathrm{p}<0.01 ; \mathrm{r}=0.45, \mathrm{p}<0.03 ; \mathrm{r}=0.46, \mathrm{p}<0.02$; and $\mathrm{r}=0.42, \mathrm{p}<0.04$, respectively), mean time $(\mathrm{r}=0.50, \mathrm{p}<0.01 ; \mathrm{r}=0.45, \mathrm{p}<0.02 ; \mathrm{r}=0.47, \mathrm{p}<0.02 ;$ and $\mathrm{r}=0.42, \mathrm{p}<0.04$, respectively), and worst sprint time $(\mathrm{r}=0.45, \mathrm{p}<0.03 ; \mathrm{r}=0.41, \mathrm{p}<0.04 ; \mathrm{r}=0.44, \mathrm{p}<0.03$; and $\mathrm{r}=0.45, \mathrm{p}<0.02$, respectively) in high intensity exercise. However, there was no relation between accuracy and body sway parameters or between high intensity exercise parameters and body sway parameters after high intensity exercise.

\section{Discussion}

The aim of this study was to investigate the effects of high intensity exercise (all-out sprints) on body sway in under-19 basketball players during bipedal stance and to correlate body sway parameters and free throw accuracy. The main finding of our study was the increased body sway after high intensity exercise, which indicated worse postural control due to the exercise, but with no effects on performance (accuracy in free throws). In addition, the results indicated a negative moderate relationship between body sway and accuracy and a positive moderate relationship between body sway parameters and performance in high intensity exercise before high intensity exercise, which was not confirmed after high intensity exercise. Therefore, our hypothesis was partially confirmed. Based on our results, in the next paragraphs we will discuss explanations and offer interpretations for: i) effects of high intensity exercise on body sway, and the possible consequences for sport performance; ii) lack of effects of high intensity exercise on free throw performance (accuracy); and iii) lack of relationship between body sway and performance after high intensity exercise.

High intensity exercise increased body sway in under-19 basketball players. The effects of high intensity exercise on postural control are, mainly, due to alterations in the sensory information from the proprioceptive and exteroceptive systems (Lepers, Bigard, Diard, Gouteyron, \& Guezennec, 1997; Paillard, 2012). In fact, initially or in no-high intensity exercise, the central nervous system manages to partially or completely compensate for the disturbance to postural function by the integration of different sensory information (Paillard, 2012). For example, the disturbance of a sensory channel (i.e., proprioception) can be compensated for by the intervention of another sensory channel (i.e., vision). However, the effects after high intensity exercise are huge, which cannot be compensated for and postural control is thus disturbed. In addition, our high intensity exercise performed through the RSA protocol mobilized general muscular activity, which amplifies liquid movements and cardiac and respiratory muscular contractions, causing 
an increase in body sway (Bove et al., 2007; Harkins, Mattacola, Uhl, \& Malone, 2005). Static postural control is essential in free throwing performance (Hudson, 1985) and increased static body sway is related to reduced accuracy (performance), injuries, and performance in exercise (Steinberg et al., 2015; Zemková, 2009; Zemková \& Hamar, 2014). Low anterior-posterior and medio-lateral body sway reduces the demand of the postural control system (Hudson, 1985), which requires less adjustments of lower limb joints, principally hip and ankle muscles. Therefore, basketball players can improve the motor control of free throwing. However, our results demonstrated no effects of high intensity exercise on accuracy, which seems to indicate that the increase in body sway did not decrease performance.

Despite impairments in postural control from high intensity exercise, the basketball free throwing performance (accuracy) was not affected. Previous studies indicate that basketball athletes seem to react to all-out exercise through decreasing accuracy (Lyons et al., 2006; Thomson, Watt, \& Liukkonen, 2009). However, our findings did not show this effect. Three aspects need to be considered to explain this finding. First, the protocol used high intensity exercise. The effects of exercise on sport performance and postural control are exercise protocol-dependent (Paillard, 2012; Zemková \& Hamar, 2014). Our high intensity exercise protocol was a general muscular exercise, which reflects the type of muscular activities encountered in basketball games (Padulo, Attene et al., 2015; Padulo, Laffaye et al., 2015; Zagatto et al., 2017). Even though this protocol could have caused central effects, the main muscles used for basketball free throwing (i.e., arm muscles) were not fatigued. Therefore, the neuromuscular system may be able to adjust, according to the deficits caused by high intensity exercise, the activity of the muscles, thus, not affecting basketball free throwing accuracy (Boyas et al., 2011; Dickin \& Doan, 2008), although it is worth remembering that lower limb muscle activity stabilizes posture during basketball free throwing, which could increase body sway. Second, the sub-maximal demands characteristic of basketball free throwing. Trained basketball players are capable of dealing with the possibly negative effects of high intensity exercise on submaximal performances that require relatively less strength but more coordination, such as free throwing (Hudson, 1982; Woolstenhulme, Griffiths, Woolstenhulme, \& Parcell, 2006). In addition, basketball free throwing is a common task for basketball athletes, which facilitates control of the postural demand and, consequently, may not present decreased accuracy even after high intensity exercise. Therefore, more complex tasks (long-range throws), such as 3-point shots, jump shots or shots against an opponent, could present decreased accuracy after high intensity exercise. Erčulj and Supej (2009) found adjustments in basketball specific movements after high intensity exercise in 3-point shots when compared with shots performed with no exercise. Third and final, freeshooting is a different task compared to quiet stance. Basketball free throwing requires control of posture and the ball. Therefore, any inaccurate adjustment in postural control could affect free throwing. Thus, we recommend that future studies investigate postural control after high intensity exercise during free throwing.

High intensity exercise inhibited the relationship between body sway and accuracy and body sway and performance in high intensity exercise. Our results indicated a relationship (moderate) between lower body sway and better accuracy and performance in exercise before high intensity exercise and, contrary to our expectations; this relationship was not found after high intensity exercise. Considering the results for accuracy, trained basketball players seem to be able to adjust (or maintain) accuracy when they are fatigued or after all-out exercise, as found by Lyons et al. (2006) for passing ability. In addition, our findings corroborate a previous study that indicated rifle shot accuracy is not affected by increased postural sway in high-level players (Era, Konttinen, Mehto, Saarela, \& Lyytinen, 1996). Moreover, the lack of relation between body sway and performance in high intensity exercise after all-out exercise indicated that aerobic intensity exercise deteriorated postural control, corroborating with Steinberg et al. (2015). Therefore, our participants seemed to deal well with increased body sway and maintain accuracy, which suggests that trained athletes can recover sufficiently in a very short period to maintain free-throwing performance.

Even with consistent and relevant results, this study has a few limitations. The present study was limited to the number of shot trials in a set (3). To measure the effects of exercise on a skill, it is better to analyse several measures of the skill. A similar limitation is verified for the quiet standing task. Future studies should consider this limitation and increase the number of free throws and the time in the standing task, however we believe this did not strongly affect our results, although the conclusion should be analysed with care. Second, the use of a zero-sum marking scheme was not used to analyse the performance. A score scheme such as 3 points for a swish, 2 for scoring off the rim or backboard, and 1 for hitting the rim but failing to score could allow differentiation in the performance. We recommend using this analysis for basketball free throwing accuracy in future studies. Third, when a postural test is performed it is common to place a target in front of the individual, positioned at a fixed distance to decrease the influence of vision on body sway. The presence of the same target at the same distance assures that everyone performs the same goal. However, we performed the postural test on the court with the force plate at the free throw line and did not place a target in front of the individuals. We chose this protocol to have no rest between the free throwing and postural test. Fourth, heart rate is a proxy of the metabolic demand. Future studies should measure this parameter during the protocol. Fifth, these data are specifically for younger basketball players. For professional basketball players, the effects of high intensity exercise on body sway should be examined. Finally, the reliability of the measures was not access in this experiment Therefore, generalization of the results should be performed with care.

\section{Conclusion}

In conclusion, high intensity exercise increased body sway in under-19 basketball players, but with no effects on basketball free throwing accuracy. In addition, no relationship was observed between body sway and accuracy, and body sway and performance in all-out exercise after high intensity exercise. 


\section{References}

Bove, M., Faelli, E., Tacchino, A., Lofrano, F., Cogo, C. E., \& Ruggeri, P. (2007). Postural control after a strenuous treadmill exercise. Neuroscience Letters, 418, $276-281$. Boyas, S., Remaud, A., Bisson, E. J., Cadieux, S., Morel, B., \& Bilodeau, M. (2011). Impairment in postural control is greater when ankle plantarflexors and dorsiflexors are fatigued simultaneously than when fatigued separately. Gait\& Posture, 34, 254-259.

Dickin, D., \& Doan, J. (2008). Postural stability in altered and unaltered sensory environments following fatiguing exercise of lower extremity joints. Scandinavian Journal of Medicine \& Science in Sports, 18, 765-772.

Era, P., Konttinen, N., Mehto, P., Saarela, P., \& Lyytinen, H. (1996). Postural stability and skilled performance-a study on top-level and naive rifle shooters. Journal of Biomechanics, 29, 301-306.

Erčulj, F., \& Supej, M. (2009). Impact of fatigue on the position of the release arm and shoulder girdle over a longer shooting distance for an elite basketball player. The Journal of Strength \& Conditioning Research.

Fitzsimons, M., Dawson, B., Ward, D., \& Wilkinson, A. (1993). Cycling and running tests of repeated sprint ability. Australian Journal of Science and Medicine in Sport, $25,82-87$.

Harkins, K. M., Mattacola, C. G., Uhl, T. L., \& Malone, T. R. (2005). Effects of 2 ankle fatigue models on the duration of postural stability dysfunction. Journal of athletic training, 40, 191.

Hebert, J. R., Corboy, J. R., Manago, M. M., \& Schenkman, M. (2011). Effects of vestibular rehabilitation on multiple sclerosis-related fatigue and upright postural control: a randomized controlled trial. Physical Therapy.

Horak, F. B., \& Macpherson, J. M. (2011). Postural orientation and equilibrium. Comprehensive Physiology.

Hudson, J. L. (1982). A biomechanical analysis by skill level of free throw shooting in basketball. Biomechanics in Sports, 95-102.

Hudson, J. L. (1985). Prediction of basketball skill using biomechanical variables. Research Quarterly for Exercise and Sport, 56, 115-121.

Lepers, R., Bigard, A. X., Diard, J.-P., Gouteyron, J.-F., \& Guezennec, C. Y. (1997). Posture control after prolonged exercise. European Journal of Applied Physiology and Occupational Physiology, 76, 55-61.

Lyons, M., Al-Nakeeb, Y., \& Nevill, A. M. (2006). The impact of moderate and high intensity total body fatigue on passing accuracy in expert and novice basketball players. Journal of Sports Science and Medicine.

Milioni, F., Redkva, P. E., Barbieri, F. A., \& Zagatto, A. M. (2017). Six weeks of $\beta$-alanine supplementation did not enhance repeated-sprint ability or technical performances in young elite basketball players. Nutrition and Health. http://dx.doi.org/10.1177/0260106017700436 in press.

Mononen, K., Konttinen, N., Viitasalo, J., \& Era, P. (2007). Relationships between postural balance, rifle stability and shooting accuracy among novice rifle shooters. Scandinavian Journal of Medicine \& Science in Sports, 17, 180-185.

Padulo, J., Attene, G., Migliaccio, G. M., Cuzzolin, F., Vando, S., \& Ardigò, L. P. (2015). Metabolic optimisation of the basketball free throw. Journal of Sports Science and Medicine, 33, 1454-1458.

Padulo, J., Laffaye, G., Haddad, M., Chaouachi, A., Attene, G., Migliaccio, G. M., ... Pizzolato, F. (2015). Repeated sprint ability in young basketball players: One vs. two changes of direction (Part 1). Journal of Sports Science and Medicine, 33, 1480-1492.

Paillard, T. (2012). Effects of general and local fatigue on postural control: a review. Neuroscience \& Biobehavioral Reviews, 36, $162-176$.

Pau, M., Ibba, G., \& Attene, G. (2013). Fatigue-induced balance impairment in young soccer players. Journal of Athletic Training, 49, $454-461$.

Perrin, P., Perrin, C., Courant, P., Bene, M., \& Durupt, D. (1990). Posture in basketball players. Acta oto-rhino-laryngologica Belgica, 45 , 341-347.

Platzer, H.-P., Raschner, C., \& Patterson, C. (2009). Performance-determining physiological factors in the luge start. Journal of Sports Science and Medicine, 27, $221-226$.

Ruscello, B., Tozzo, N., Briotti, G., Padua, E., Ponzetti, F., \& D'Ottavio, S. (2013). Influence of the number of trials and the exercise to rest ratio in repeated sprint ability, with changes of direction and orientation. The Journal of Strength \& Conditioning Research, 27, 1904-1919.

Steinberg, N., Eliakim, A., Zaav, A., Pantanowitz, M., Halumi, M., Eisenstein, T., ... Nemet, D. (2015). Postural balance following aerobic fatigue tests: A longitudinal study among young athletes. Journal of Motor Behavior, 1-9.

Steinberg, N., Nemet, D., Pantanowitz, M., Zeev, A., Hallumi, M., Sindiani, M., ... Eliakim, A. (2016). Longitudinal study evaluating postural balance of young athletes. Perceptual and Motor Skills, 122, 256-279.

Thomson, K., Watt, A., \& Liukkonen, J. (2009). Differences in ball sports athletes speed discrimination skills before and after exercise induced fatigue. Journal of Sports Science and Medicine, 8, 259-264.

Uygur, M., Goktepe, A., Ak, E., Karabörk, H., \& Korkusuz, F. (2010). The effect of fatigue on the kinematics of free throw shooting in basketball. Journal of Human Kinetics, 24, 51-56.

Winter, D. A. (2009). Biomechanics and motor control of human movement. John Wiley \& Sons.

Woolstenhulme, M. T., Griffiths, C. M., Woolstenhulme, E. M., \& Parcell, A. C. (2006). Ballistic stretching increases flexibility and acute vertical jump height when combined with basketball activity. The Journal of Strength \& Conditioning Research, 20, 799-803.

Zagatto, A. M., Ardigò, L. P., Barbieri, F. A., Milioni, F., Iacono, A. D., Camargo, B. H., \& Padulo, J. (2017). Performance and metabolic demand of a new repeatedsprint ability test in basketball players: Does the number of changes of direction matter? The Journal of Strength \& Conditioning Research. http://dx.doi.org/10. 1519/JSC.0000000000001710 in press.

Zemková, E. (2009). Balance readjustment after different forms of exercise: a review. International Journal of Sports Science, 21, 45-60.

Zemková, E., \& Hamar, D. (2014). Physiological mechanisms of post-exercise balance impairment. Sports Medicine, 44, 437-448. 